\title{
SCHNITTSTELLEN VON ERFAHRUNG UND ERWARTUNG. EIN PLÄDOYER FÜR STORYS IM INTERKULTURELLEN LITERATURUNTERRICHT
}

\begin{abstract}
Interfaces between experience and expectation. A plea for stories in intercultural literature education

The specific situation of a German academic working outside of Germany emerges via the differential and non-simultaneous absorption (or incorporation) of methodological schools and traditions. This article shows a possible means of executing the balancing act between the learned "third culture" and your own tradition. It is about the interfaces between experience and expectation which one can find them through understanding the narrative structures. One can glean profound understanding via: narrative structure, deep structure of the text, on the basis of stories and cultural tropes, and through to structured narrative itself and to the story than the way the literature appropriation in the foreign language specific perspective. We only know as much as we are able to express. The language cultural identity exists because of the language and it is equal as narrative identity.
\end{abstract}

KEY WORDS: experience and expectation, intercultural literature education, narrative structures, narrative identity, narrative psychology.

Interkultureller Literaturunterricht an den Universitäten stößt unvermeidlich auf das Problem der kulturbedingten (Multi-)Variabilität des Literaturverstehens und der Literaturinterpretation. Beide Vorgänge, sowohl das Literaturverstehen als auch die Literaturinterpretation, sind bedingt durch die von den Studierenden in den Unterricht „mitgebrachten“ Erfahrungen. Ohne Erfahrung, im Sinne eines Vorwissens, ist das Interpretieren nicht möglich. Aus den „mitgebrachten“ und den im Laufe des Studiums gesammelten Erfahrungen ergeben sich die Erwartungen der Studierenden. Um das Zusammenspiel von Erfahrung und Erwartung gerade im interkulturellen Literaturunterricht zu vertiefen, ist es sinnvoll, die zwei von Reinhart Koselleck für die Begriffsgeschichte geprägten Kategorien „Erfahrungsraum“ und „Erwartungshorizont“ im Blick zu behalten. Es gibt keine Vergangenheit, so Koselleck, „ohne daß sie durch Erfahrungen und Erwartungen der handelnden Menschen (...) konstituiert 
worden wäre“ (Koselleck, 1979, S. 351). Erfahrung sei „gegenwärtige Vergangenheit, deren Ereignisse einverleibt worden sind und erinnert werden können“ (ebd., S. 354-355).

Den Kern der Spannung zwischen dem Vergangenen und dem Kommenden drückt anschaulich ein Vers aus dem Buch Kohelet aus: „Was geschehen ist, wird wieder geschehen (...). Es gibt nichts Neues unter der Sonne“ (Koh 1,9). Ein solcher Blick auf kleinere Kommunikationsgemeinschaften, wie z. B. eine Seminargruppe von Studenten, die sich als Ausländer mit der deutschen Literatur befassen, also aus einem anderen Kulturkreis kommen als der, der zum Gegenstand ihrer Studien wird, eröffnet differenzierte Perspektiven und Übergänge von der akademischen Theorie in die berufliche Praxis. Für das interkulturelle Lernen im Rahmen der Auslandsgermanistik und des germanistischen Literaturunterrichts könnte hier ein möglicher Weg vorgeschlagen werden, der das ursprüngliche, ins Studium mitgebrachte Kommunikationssystem der Lernenden nicht ruhen lassen würde, sondern die Bikulturalität - also das ,doppelte“ Verhältnis zwischen der in den Lernprozess mitgebrachten Kultur und der zu lernenden bzw. bereits gelernten Kultur anerkennt und fördert (Karolak, 1999, S. 9-10). Es geht also darum, die Kompetenzen des Studierenden so anzuregen, dass er sich selbst mit den Augen der anderen wahrzunehmen vermag. Über die Anderen zu lernen und sich die andere Kultur anzueignen, sollte einen besonderen Gewinn bedeuten, eine einmalige Möglichkeit sein eigenes „Ich“ neu zu gewinnen (vgl. Mead, 1975; nach: Karolak, 1999, S. 45). Da jede Kultur nicht anders als nur im Kontrast entsteht, kann sie sich selbst insoweit wahrnehmen, als sie das „Andere“ und „Fremde“ zu erfahren fähig ist. Wird aber das Erwartete zum Erfahrenen, um wieder mit Reinhart Koselleck zu sprechen, so entsteht eine neue, dritte Kultur, eine neue Qualität, die aus dem Ineinandergehen der Ausgangskultur und der erlernten, angeeigneten Kultur besteht (Karolak, 1999, S. 43) und sich in den mitgebrachten Erfahrungsraum, der dank der Lernneugier neue Erwartungshorizonte eröffnet, einnisten kann. Diese dritte Kultur wird zu einer Bereicherung, zu einem Zuwachs und Perspektivenwechsel, zu einer neuen Selbstwahrnehmung und einer einmaligen Chance für die Lernenden.

In diesem Sinne, zusammen mit der Aneignung einer fremden Kultur, kann auch der Prozess der Herausbildung einer neuen ,dritten“ Identität beginnen, was eine Herausforderung und eine besondere Aufgabe für den ausländischen Germanistikstudenten darstellen sollte. Die spezifische Situation des fremdsprachigen Literaturunterrichts zeigt sich in der unterschiedlichen und ungleichzeitigen Absorption (oder Inkorporation) methodologischer Schulen und Traditionen. Es wird erwartet, dass der Lernende, mit einem methodologischen Vorwissen aus dem Unterricht in der Oberschule ausgestattet, im Laufe des Germanistikstudiums die deutsche literaturwissenschaftliche Methodologie erlernt und dabei parallel, im Grunde virtuell, den Sozialisierungsprozess seiner gleichaltrigen deutschen Muttersprachler „,nachholt“; und dann, als ausgebildeter Germanist, in seinem beruflichen Leben (im besten Fal- 
le) weiterhin den methodologischen Stand (wenigstens) in den deutschsprachigen Ländern verfolgt, ohne die Diskurse im eigenen Land zu verpassen. Für diesen $\mathrm{Zu}$ stand der neuen Sozialisierung, des Nachholens und des Sichbewegens in zwei parallelen intellektuellen Kreisläufen, prägte der polnische Germanist Hubert Orłowski die Bezeichnung der „doppelten Nabelschnur“ als Prinzip, dem der Auslandsgermanist unterlegen ist (vgl. Orłowski, 1987, S. 119). Und es ist verständlich, dass dieser Kreislauf durch seine doppelte Laufbahn belastet ist und daher langsamer gehen muss, was aber bei Weitem nicht heißt, dass er deswegen im Weniger endet - sondern in einem Mehr. Orłowskis bildhafte Darstellung des doppelten Kreislaufs könnte mit zwei Aneignungswegen des Wissens verglichen werden: dem Weg des bisher Erfahrenen und dem des zu Erwartenden.

Im Folgenden soll der Versuch unternommen werden, Aneignungswege zu zeigen, die im didaktischen Prozess beide Kreisläufe zugleich betreffen und damit Möglichkeiten verschaffen, dass beide Nabelschnüre „zusammenwachsen“ und der Spagat zwischen der „dritten Kultur“ und dem verlangsamten Kreislauf der doppelten Nabelschnur gelingen kann. Es sind die Schnittstellen von Erfahrung und Erwartung, der mitgebrachten bisherigen Kultur und der angelernten Kultur; Stellen, an denen beide Kreisläufe vereint sein könnten. Es scheint überlegenswert zu sein, ob und wie sie im fremdsprachigen Literaturunterricht zu erreichen sind.

Diese Verbindungsstellen können über Wege führen, die in unterschiedlichen Kulturen ähnlich verlaufen. Es sollten hier nur beispielhaft drei von vielen möglichen genannt werden. Eine Möglichkeit bietet das tiefgründige Verständnis der Erzählstrukturen und der Tiefenstrukturen der Texte, eine andere die Basiserzählungen (Basic-Storys oder Basic-Plots) ${ }^{1}$ bis hin zum strukturierten Erzählen selbst und zur Story als DEM Weg der Literaturaneignung in der fremdsprachenspezifischen Perspektive im interkulturellen Unterricht.

In den Erzählstrukturen, die unabhängig von der Sprache immer dieselben sind, sind identitätsstiftende Repräsentationen enthalten. Mit diesen Strukturen sind hier Muster gemeint, die der Weltliteratur, dem Erzählten überhaupt, zugrunde liegen. Insbesondere werden sie in den alltäglichen Erzählungen sichtbar: in den Werbespots, in den Nachrichten, in den Presseartikeln. Die Aufdeckung und Wahrnehmung der dem alltäglichen Erzählen zugrunde liegenden universellen Grundmuster bzw. Schemata erlaubt den Lernenden, das Gefühl einer guten Textorientierung zu gewinnen, sogar wenn sie sprachlich noch nicht fähig sind, jedes Wort aus dem fremdsprachigen Text zu verstehen. Das Erkennen der Darstellungsmechanismen, wie: der Ausgangslage eines Textes, der Mittel, mit denen die Textabweichung und dann auch ein entsprechendes Ende konstruiert werden (vgl. Nusser, 1991, S. 119-129),

\footnotetext{
${ }^{1}$ Der Begriff „Basiserzählung“ wird hier im Sinne der Narration (insbesondere Storytelling) verwendet. Es gibt eine differenzierte Bedeutung dieses Begriffs, insbesondere im Kontext der NS-Vergangenheit und der Forschung zu kollektiven Gedächtnissen, geprägt u. a. von Thomas A. Herz (1997).
} 
ist eine Grundfertigkeit, die einen fremdsprachigen Text, eine Story, eine literarische Form schnell zu überblicken erlaubt. Auch die Darstellungsstrategien bzw. Darstellungskonventionen gehören in die Sparte der die Textwahrnehmung erleichternden Grundschemata: die Strategie der Personalisierung, die die Probleme simplifizierend in Personen verdichtet, oder die ihre Identifikation erleichternde Typisierung der Figuren oder einfach die Bestätigung von Erwartungen der Leser und zweckmäßige Steuerung der Spannung in den erzählten Geschichten (Nusser, 1991, S. 119-129). All das sind, dem ersten Anschein nach, Raffinessen, die eigentlich ein Angebot an universellen Vereinfachungen enthalten, an unvermeidlichen Werturteilen und wirksamen Strukturen, die die Wirklichkeit überschaubar machen. Im Literaturunterricht bei der Textanalyse geschickt aufgedeckt, können sie als die wenigen, doch entscheidenden Momente bezeichnet werden, in denen beide Welten der Lernenden, ihre Erfahrung und ihre Erwartung, zusammenkommen, in denen die bisher getrennt verlaufenden Nabelschnüre plötzlich eins werden.

Eine andere Möglichkeit der „Verschmelzung“ beider Kreisläufe bei einem Lernenden ist in den sog. Basiserzählungen (Basic-Stories) zu suchen. Archetypen, Mythen, Märchen sind Manifestationen der menschlichen Seele, die nicht unbedingt kulturell gebunden sein müssen. Würde man die Frage stellen, wie viele Basic-Stories von der Menschheit erfunden wurden, Millionen oder Tausende, so wäre es möglich eine Antwort zu finden, die sogar auf eine konkrete Zahl hinweisen würde. Es gibt eine Fülle von theoretischen Konzepten, die die Zahl dieser „Basic-Plots“ unterschiedlich bestimmen. Eines der vielen Beispiele mag der von Christopher Booker, dem englischen Journalisten und Autor, veröffentlichte Überblick über die Grundmuster der Geschichten sein: The Seven Basic-Plots: Why We Tell Stories (2005). Während Booker von sieben Basiserzählungen ausgeht, schlägt Raymond G. Frensham, der englische Autor von Bestsellern über Screenwriting, mit guter Begründung vor, acht Basic-Stories zu unterscheiden: Achill, Aschenputtel, Candide, Faust, Kirke, Orpheus, Romeo und Julia, Tristan. Frensham meint, alle acht Basiserzählungen würden millionenfach als Tragödien, Komödien bzw. Krimis neu erzählt, indem sie nur unterschiedlich miteinander gemischt, aneinander angepasst, sogar auf den Kopf gestellt werden und doch immer Fundament und Basis aller Storys bleiben (Frensham 1997, S. 57); und dies unabhängig von jeder Sprache und Kultur. Frensham argumentiert, jede erzählte Geschichte lasse sich letztendlich auf ihren Kern reduzieren und auf eine der wenigen Urgeschichten bzw. Basiserzählungen zurückführen. Diese Vereinfachung, aber zugleich auch Essenz aller Geschichten, die die Menschheit bewegen, ist besonders leicht in zahlreichen Werbespots zu erkennen. Diese sprechen Emotionen an, indem sie sich allgemein verständlicher Basiserzählungen bedienen, eben der u. a. von Frensham aufgezählten. Nicht selten sind es Werbespots, die in mehreren Ländern parallel ausgestrahlt werden, die unverändert dieselben menschlichen Erfahrungen ansprechen und damit auch dieselben Erwartungen erzeugen. Mit Hilfe dieser Geschichten bzw. Storys wird erfolgreich verkauft. Dieses dem Verkauf untergeordne- 
te Erzählen hat heute schon seinen professionellen und anerkannten Namen gefunden, nämlich „Strateging Narrative“ oder „Narrative Marketing“. „Geschichten, die verkaufen“, die ihren Ursprung in der Literatur haben, sprechen unverändert intensiv dieselben Emotionen der potenziellen Käufer in unterschiedlichen Ländern und Kulturen an. Im interkulturellen Literaturunterricht können diese Geschichten einen Ausgangspunkt für das Erkennen der Grundmuster in verschiedenen Texten bilden.

Längst haben Psychologen nachgewiesen, dass wir nur so viel von uns selbst wissen, wie wir ausdrücken können. Es sind vor allem der französische Philosoph Paul Ricoeur, aber auch andere, u. a. der kanadische Denker Charles Taylor, die die sprachbedingte kulturelle Identität mit der narrativen Identität gleichsetzten. Die narrative Identität wird von ihnen als die zentrale Form der Selbstinterpretation verstanden. Paul Ricoeur sieht die Narration als den kulturellen Kode, der uns unsere Erfahrung zu formen und zu verstehen erlaubt (Ricoeur, 1988, 1989, 1991). Nach Charles Taylor können wir dank dem, was ausgesprochen wird, vom Unbestimmten zum eindeutig Formulierten übergehen (Taylor, 2001). In den verschiedensten Bereichen der Psychologie, im Zusammenhang mit dem ,linguistic turn', werden immer stärker die sprachlichen Vermittlungsformen mit ihren zahlreichen Funktionen als zentral und entscheidend wahrgenommen und untersucht. Der Anfang dieser Denkrichtung hängt mit der Veröffentlichung des von dem amerikanischen Psychologen Theodore R. Sarbin (1911-2005) herausgegebenen Sammelbandes Narrative Psychology - The Storied Nature of Human Conduct (1986) zusammen. Sarbin war der Pionier unter den forschenden Psychologen seiner Zeit und adaptierte die Methode des Verstehens des menschlichen Verhaltens, in der das Primat der erzählten Storys als der Weg zum Menschen gewählt wurde. Diesem methodischen Ansatz der narrativen Psychologie liegt der Versuch zugrunde, Erzählungen von Menschen als eine Sinngebung zu nutzen. Man setzt voraus, dass den Ausgangspunkt der Erzählungen nicht primär Fakten bilden, sondern vielmehr die aktuelle Gegenwart des Erzählenden, die räumlich und zeitlich bedingt ist. In den letzten zwanzig Jahren hat die narrative Psychologie Anhänger aus vielen Wissensgebieten gewonnen und sich enorm weiterentwickelt. Geschichtswissenschaftler, Psychoanalytiker, auch Soziologen erforschen Möglichkeiten und Wege, wie Geschichten (Storys) bei der Verständigung über die Vergangenheit, aber auch bei der Suche nach einer Identität helfen können. Die allermeisten Wissenschaftler, die sich der narrativen Perspektive zugehörig fühlen, stimmen in der konstruktivistischen Auffassung überein, dass die Narration das primäre strukturierende Schema der Wirklichkeit sei, durch das Personen ihre Identität und ihr Verhältnis zur Umwelt definieren und mit Sinn und Bedeutung füllen. Voraussetzung hierfür ist die postmoderne Überzeugung, dass jedes Verstehen eine Konstruktion unseres Geistes darstellt ${ }^{2}$; durch äußere Einflüsse ist diese ständigen Veränderungen unterworfen. Die

\footnotetext{
${ }^{2}$ Mehr zur narrativen Konstruktion der Identität: Wolfgang Kraus (1996). Zur Bedeutung der Erzählung des eigenen Lebens und zu Formen des Selbstbezugs vgl. Dieter Thomä (1998).
} 
Vorstellung, dass Menschen ihr Leben erzählen und dadurch subjektive Bedeutungskonstruktionen schaffen, ist übrigens nicht ganz neu. Schon Sigmund Freuds Fallstudien in seinem 1900 veröffentlichten Werk Traumdeutung schilderten den Zusammenhang zwischen Träumen und der persönlichen Lebensgeschichte. Mit Erzählungen individueller Lebensläufe befassten sich nach Freud auch die beiden US-amerikanischen Persönlichkeitspsychologen Henry Murray (1938) und Gordon Allport (1955) Es liegt auch nahe in diesen Zusammenhang Pierre Bourdieus Überlegungen zur ,biographischen Illusion“ zu stellen, um mit seinen Augen die Lebensgeschichte als die Rede vom Leben als einer „Geschichte“ (Bourdieu, 1998) wahrzunehmen.

Die Verbindung zwischen der Erzähltheorie und dem Text selbst ist selbstverständlich, genauso auch wie die Erfassung der Vergangenheit als Erzählung oder auch als Story. Die Geschichte als Wissenschaft gehört auch in diese Kette von gegenseitigen Verflechtungen. Es gibt doch keinen Staat, keine Nation ohne Geschichte, ohne Tradition, ohne Mythen, die die Staats- oder Nation-Angehörigen miteinander verbinden, die den Fortschritt fördern und die Dynamik erzeugen (im Sinne von Herder, 1881, S. 43). Die eigentliche Bedeutung von Geschichten, aus denen sich DIE Geschichte - die Historie als Wissenschaft - zusammensetzt, zeigte der große deutsche Historiker Leopold von Ranke treffend, indem er meinte, dass die Historie zugleich Kunst sei. Wissenschaft sei sie zum einen, weil sie sammelt, findet, durchdringt - und Kunst zum anderen, weil sie das Gefundene, Erkannte wieder gestaltet, darstellt. Ranke sah also zwei Gesichter der Historie: das wissenschaftliche, welches der Philosophie verwandt sei, und das künstlerische, welches mit der Poesie zusammenspielt. Während sich Philosophie und Poesie im „idealen Moment bewegen“ ist die Historie „auf ein reales angewiesen“ (Ranke, 1975, S. 72). Rankes Ausführungen heben das Ideale an der Geschichte hervor, zugleich aber führen sie auf das Reale zurück. Das erzählende Ich, seine Zeit und sein Raum, machen u. a. die Realität der Geschichte aus. Es ist gerade so, wie es die narrative Psychologie meint: der Tatsache folgend, dass eben „Geschichten“ oder „Storys“ über die Welt regieren, dass jedes Land „seine Story“ unabhängig von seiner Größe oder Stärke hat (Mistewicz, 2011, S. 5), und umso mehr jeder Mensch seine Identität in Geschichten und durch Geschichten entwickelt.

Längst hat die Businesswelt entdeckt, wie wichtig „Erzählungen“, also Geschichten, für strategisches Handeln der Menschen an ihren Arbeitsplätzen sind, was Geschichten für die Sinnkonstruktion der Unternehmen und ihrer Produkte bedeuten. Der Australier Shawn Callahan, ein hervorragender Schöpfer von Anekdoten für den Aufbau von Businessnarrationen, ist überzeugt: Geschichte, als Narration aufgefasst, bilde das primäre strukturierende Schema der Wirklichkeit, das den Menschen ermöglicht, ihre Identität und ihr Verhältnis zur Umwelt zu definieren und mit Sinn und Bedeutung zu füllen (Anecdote, 2004) ${ }^{3}$. Wenn wir feststellen: „Der König stirbt

\footnotetext{
${ }^{3}$ Mehr dazu vgl. http://www.anecdote.com/ (9.07.2016).
} 
und die Königin weint“, beschreiben wir nur Fakten. Wenn wir aber sagen: „Der König stirbt und die Königin weint, weil ihr das Herz bricht" - erzählen wir schon eine Geschichte (Mistewicz, 2011, S. 56). In seinem Bestseller Tell to win schreibt Peter Guber, der US-amerikanische Filmproduzent und einflussreicher Vertreter der Unterhaltungsindustrie:

Today everyone - whether they know it or not - is in the emotional transportation business. More and more, success is won by creating compelling stories that have the power to move partners, shareholders, customers and employees to action. Simply put, if you can't tell it, you can't sell it. (Gruber, 2011, S. 13)

Das Geschäft ist heute also mit Storys zu machen - mit Storys, die Emotionen ausdrücken, die Wurzeln aufdecken und damit stabilisierend und zukunftweisend wirken. Übrigens trägt eines der Kapitel in Tell to win den Titel "The Art of the Tell", was Rankes wissenschaftlichen Überlegungen auf eine popularisierende Weise doch gerecht wird: Erzählen sei Kunst, so wie die „Historie“ Kunst ist.

Doch in der eben erwähnten Story vom Tod des Königs sieht der Schweizer Schriftsteller (und zugleich Unternehmer) Rolf Dobelli eine Gefahr, die für den interkulturellen Literaturunterricht gerade von größtem Vorteil sein könnte (Dobelli, 2011, S. 53-56). Dobelli steht Sachverhalten, die in Form von Geschichten erzählt werden, skeptisch gegenüber und weist darauf hin, dass die vermittelten Storys leicht zur Quelle eines „Denkfehlers“ werden, des sogenannten „story bias“. Geschichten verdrehen und vereinfachen die Wirklichkeit. Sie verdrängen alles, was nicht so recht hineinpassen will - so Dobelli. Der "story bias“ liege genau an der Stelle vor, wo eine Tatsache zur Story wird. Also dann, wenn die Erzählung von einer Königin, die wenige Tage nach dem Tod ihres Ehemannes gestorben ist, mit den Worten wiedergeben wird: „Der König starb, und dann starb die Königin vor Trauer". Auf der einen Seite bleiben den Lesern so vermittelte Botschaften länger im Gedächtnis, auf der anderen wird so durch das Erzählen jedoch in eine wahre Begebenheit ein neuer Sinn hineinkonstruiert, der mit der Wirklichkeit nichts zu tun hat. Dobelli meint, dies sei das Ungerechte, die Falle, in die die Geschichten unvermeidlich tappen - eben der „story bias“. Für die narrative Psychologie, aber auch sehr wohl für den Literaturunterricht, ist dies genau der Moment, wo das Kreative, das Schöpferische einsetzt, wo Fakten aufhören und Geschichten beginnen - Geschichten, das muss hinzugefügt werden, die sich ins Gedächtnis einprägen, die gut klingen und gerne gehört bzw. gelesen werden. Es ist der Moment, in dem nicht nur beide Kreisläufe eines Auslandsgermanisten erst richtig pulsieren können. Es ist zugleich eine große Chance, die Literatur an der Universität marktorientiert zu unterrichten, was bedeutet: so zu unterrichten, dass die Literaturseminare und Vorlesungen nicht nur im Studium wirksam werden, sondern es später auch erlauben, in der beruflichen Praxis ,auf dem Markt“ die gewonnenen Kompetenzen einzusetzen. Im Sinne der Verschmelzung des Realen und des Idealen an einer Geschichte. 
Einen guten Zugang zu den Basiserzählungen und zugleich eine erleichterte Erkenntnis der Erzählstrukturen bietet die nicht-kanonisierte Literatur - vor allem die Unterhaltungsliteratur und das sogenannte „triviale Buch“, das in dem Erfahrungsraum der Lernenden leicht(er) identifizierbar ist. Als Gegensatz zur hohen Literatur kann eben das „unterhaltende, triviale Buch“ ihr paradoxerweise den Weg ebnen, weil in ihm die bereits vertrauten Strukturen (die zum Erfahrungsraum gehören) leichter zu erkennen sind. Dies betrifft sowohl Lektüren von Erwachsenen als auch von Jugendlichen. Der natürliche Weg der Sozialisation eines Muttersprachlers erfolgt meistens auch zuerst durch die Strukturen der Kinder- und Jugendliteratur, später dann oft durch die der Unterhaltungsliteratur, bevor die Jugendlichen nach der hohen und anspruchsvollen, kanonisierten Literatur greifen. Die nachzuholende deutschsprachige Sozialisation eines Auslandsgermanisten könnte erfolgreicher verlaufen, wenn er sich im Studium zuerst mit der deutschen unterhaltenden Belletristik befassen würde, bevor er sich mit dem Literatur-Kanon vertraut macht und die deutschen Klassiker zu lesen beginnt. Reinhart Koselleck meint, die Vergangenheit und die Zukunft kommen niemals zur Deckung (Koselleck, 1979, S. 356), und diesem Gedanken folgend, ist es nicht unberechtigt zu behaupten, dass die beiden Nabelschnüre nie zusammenwachsen können - doch auf keinen Fall müssen sie immer nur nebeneinander existieren.

\section{Bibliographie}

Allport, G. (1955). Letters from Jenny. New York: Harcourt, Brace \& World.

Anecdote (2004): Putting stories to work. Abgerufen am 9. Juli 2016 von http://www.anecdote.com

Booker, Ch. (2005). The Seven Basic-Plots: Why We Tell Stories. Bloomsbury: Academic. Bourdieu, P. (1998). Praktische Vernunft. Zur Theorie des Handelns. Frankfurt a. M.: Suhrkamp.

Dobelli, R. (2011). The Story Bias. Warum selbst die wahren Geschichten lügen. In: Die Kunst des klaren Denkens. 52 Denkfehler, die Sie anderen überlassen sollten. München: Hanser.

Frensham, R. (1997). Screenwriting, London: Hodder \& Stoughton. Polnische Ausgabe: Jak napisać scenariusz (1998). Kraków: Literackie.

Frensham, R. (2011). Brilliant Screenwriting. London: Hodder Education.

Freud, S. (1972). Die Traumdeutung. Studienausgabe Bid. 2, Frankfurt a. M.: Fischer.

Gruber, P. (2011). Tell to win. Connect, Persuade and Triumph with the Hidden Power of Story. New York: Crown Business.

Herder, J. G. (1881). Briefe zur Beförderung der Humanität. Sämmtliche Werke Bd. 17. Berlin: Weidmann.

Herz, T. A. (1997). Die „Basiserzählung“ und die NS-Vergangenheit. Zur Veränderung der politischen Kultur in Deutschland. In T. A. Herz \& M. Schwab-Trapp (Hrsg.), Umkämpfte Vergangenheit. Diskurse über den Nationalsozialismus seit 1945 (S. 249-266). Opladen: Westdeutscher Verlag.

Karolak, Cz. (1999). Dydaktyka literatury wobec potrzeb nauki języka w warunkach obcokulturowych. Poznań: Naukowe UAM.

Koselleck, R. (1979). Vergangene Zukunft. Zur Semantik geschichtlicher Zeiten. Frankfurt a. M.: Suhrkamp. 
Kraus, W. (1996). Das erzählte Selbst. Die narrative Konstruktion von Identität in der Spätmoderne. Pfaffenweiler: Centaurus.

Mead, G.H. (1975). Geist, Identität und Gesellschaft. Frankfurt a. M.: Suhrkamp.

Mistewicz, E. (2011). Marketing narracyjny. Gliwice: Helion.

Murray, H. (1938). Explorations in Personality. A Clinical and Experimental Study of Fifty Men of College Age. New York: Oxford University Press.

Nusser, P. (1991). Trivialliteratur. Stuttgart: Metzler.

Orłowski, H. (1987). Die doppelte Nabelschnur fremdsprachlicher Germanistik. In A. Wierlacher (Hrsg.), Perspektiven und Verfahren interkultureller Germanistik. (S. 113-124). München: Iudicium.

von Ranke, L. (1975). Idee der Universalhistorie. In V. Dotterweich \& W. P. Fuchs (Hrsg.), Vorlesungseinleitungen. Aus Werk und Nachlass, Bd. 4. (S. 72). München: Oldenbourg.

Riceur, P. (1988). Zeit und Erzählung, Bd. 1: Zeit und historische Erzählung. München: Fink.

Riceur, P. (1989). Zeit und Erzählung, Bd. 2: Zeit und literarische Erzählung. München: Fink.

Riceur, P. (1991). Zeit und Erzählung, Bd. 3: Die erzählte Zeit. München: Fink.

Sarbin, T. R. (1986). (Hrsg.) Narrative Psychology - The Storied Nature of Human Conduct. New York: Praeger.

Taylor, Ch. (2001). Źródła podmiotowości. Narodziny tożsamości nowoczesnej. Warszawa: PWN.

Thomä, D. (1998). Erzähle dich selbst. Lebensgeschichte als philosophisches Problem. München: Beck. 